\title{
ELEMENTOS PARA UNA PROSPECTIVA DE LA UNIVERSIDAD COLOMBIANA EN LA SOCIEDAD DEL CONOCIMIENTO
}

Alhim Adonaí Vera Silva: Universidad Surcolombiana (Colombia)

"Los jóvenes de una Nación

Son los depositarios de

La posteridad"

Benjamín Disraeli

\section{Introducción}

En el presente artículo se analizan las relaciones entre las normas concebidas desde la burocracia que amarran el futuro de las universidades públicas a sus intereses particulares representados en los Consejos Superiores, a través, de por lo menos dos figuras: la discrecionalidad y la delegabilidad en la Sociedad del Conocimiento que nos invita a construir, desde la prospectiva, los escenarios de las universidades del futuro, dónde, las normas arropan el ejercicio de la democracia en la transformación positiva del bienestar social.

El argumento central de esta tesis considera que los actores de la universidad conciertan los escenarios futuros de la universidad a través de colectivos; convierten en normas las decisiones asumidas por los actores; establecen los requisitos, condiciones y compromisos económicos, científicos, tecnológicos y académico administrativos para la transformación con el concurso de los distintos actores políticos, académicos, culturales, sociales y productivos.

La reflexión en torno al papel de los actores universitarios en la transformación de la universidad tiene que ver con la relación entre la visión prospectiva de la universidad, las nuevas normas que la regulan y el compromiso científico, político, económico social y cultural que deben asumir los actores para concertar la transformación de la universidad.

En Colombia no ha nacido el nuevo proyecto de Universidad del futuro y las disquisiciones de los distintos sectores de la sociedad giran en torno a cómo construirle a la universidad una jaula normativa, que, inmovilice su trasformación a través de capítulos y una serie de artículos que controlen, amarren, frenen, castiguen e impidan que la universidad se escape del dominio del Estado Burocrático. 
Los retos, la innovación, lo nuevo, la crisis, nos produce inmovilidad que solucionamos con la norma burocrática, con la magia reguladora de lo escrito; qué tantos 'palitos' debe tener la jaula inmovilizadora del futuro, unos cuantos artículos por aquí, otros por allá un 'paragrafito' para que la discrecionalidad se haga visible; unos incisos para la delegabilidad mutante; unas trancas más allá para los académicos audaces que les da por pasar la frontera legal, unos mataburros en aquella curva que permita eliminar aspectos indeseables competitivos, ¿qué tal les parece un articulado sobre la imagen simbólica del rectorable?, unos requisitos escritos que coincidan con los intereses políticos, económicos o sociales de los grupos dominantes de la sociedad. Qué hábiles somos nosotros para conservar, lo viejo por lo viejo, o por su potencial de prebendas y qué frágiles somos para abordar la complejidad del futuro a través de la visión de sus escenarios que debe asumir la universidad en el siglo XXI.

En el nuevo escenario de la Sociedad del Conocimiento la norma tradicional -que funcionó para consolidar el desarrollo de la economía de los poderosos en la sociedad esclavista griega, en la sociedad romana discriminatoria, en la oscurantista feudal, o en la capitalista salvaje-, está en crisis por su incapacidad de regular las relaciones de poder en la nueva Sociedad del Conocimiento. La fragilidad de la dimensión normativa tradicional referida a los estados actuales está descrita en una bella paradoja de Carlos Tünermann cuando afirma "El estado actual es demasiado grande para las pequeñas cosas y demasiado pequeño para las grandes cosas", estamos frente a un estado incapaz de regular el futuro de la universidad, desde el discurso normativo, y menos aún, proyectarlo a través de la discrecionalidad de la delegabilidad que le han otorgado a su órgano de poder: "El Consejo Superior Universitario" en las universidades públicas.

La tradicional composición de los órganos del poder de una organización universitaria pública regulado por la norma tiene un significado oculto que la relaciona con las mismas concepciones de poder y de organización que manejan las burocracias en sus partidos tradicionales y en los órganos del Estado, bajo dos figuras "delegabilidad" y "discrecionalidad". Las formas tradicionales de la articulación de los partidos en la toma de decisiones tienen relaciones con estas dos figuras que adquieren su cuerpo en el instante del voto. Con relación a la "delegabilidad" los delegados los hay de tantas formas: edad, categoría, tinte, atractivo, fuerza y pedigrí, dependiendo del poder con que ha sido ungido el delegado, para que lo juegue en la mesa de los intereses, o fuera de la mesa, o debajo de la mesa; incluso, es común encontrar delegado del delegado. Suplente que se expresan en funciones sui géneris como; delegado emergente, delegado suplente, delegado incógnito, delegado silencioso; algunos transforman los leyes de la materia y adquieren el don de la ubicuidad. Lo fascinante de este fenómeno de la delegabilidad, es que el delegado puede aparecer y desaparecer por arte de magia en el momento de las decisiones de acuerdo a los intereses que sirve. 
En relación con la discrecionalidad, los consejos superan las mismas concepciones deprimentes que manejan las burocracias tradicionales de los partidos, parece que no es vital el capital cultural, en palabras Bourdieu, de los actores universitarios para construir el nuevo ethos académico de la universidad; por ello quizás se explica, la fragilidad de ciertas paridades numéricas o ciertos coeficientes matemáticos que dan "legitimidad" o "deslegitimidad" a las decisiones a través de unos numerillos, a los cuales se les puede estirar o encoger el pescuezo cuando, por ejemplo, de elegir a un rector se trata; lo importante es que el elegido quepa dentro del hueco normativo que la discrecionalidad y la delegabilidad que el "Concejo" le ha propiciado para su elección o rechazo.

Desde esta perspectiva, es fundamental estudiar con rigurosidad científica, el grado de las contradicciones que el manejo del poder ha generado como símbolo de intromisión de los partidos tradicionales y del Estado, en el fenómeno llamado universidad y sus efectos en la construcción del futuro de la universidad. Crece como verdolaga la arbitrariedad de la burocracia que vulnera la "autonomía" de las universidades y fortifica a los estrados judiciales que deslegitiman en horas lo que le costó a la universidad construir en siglos

En los escenarios del siglo XXI, las ideas las "Honorables Miembros del consejo Universitario (HCSU) deben provenir de las personas más idóneas, éticas, morales y sabias de la sociedad del conocimiento con experticia en asuntos internacionales, nacionales y regionales y locales aunado al aporte de pergaminos académicos que muestre senderos en la construcción de la universidad del siglo XXI. Sus figuras académicas infunden respetabilidad social, admiración científica y liderazgo organizacional, reunidas en diálogos recreativos y prospectivos de las mentes más preclaras, más lúcidas, más brillantes más connotadas del país de la región; creando, proponiendo, construyendo, bocetando, gestionando, generando ambientes y escenarios para la maduración de las ideas; propiciando las innovaciones las estrategias de trabajo; la modernización de la infraestructura científico tecnológica; gestionando fuentes de financiación y cooperación regional, nacional e internacional para los proyectos de la universidad del futuro. Un encuentro del saber, abierto a los medios de comunicaciones, en transmisión directa televisada e interactiva, como ya lo hacen el Congreso de la Republica, el Senado y la misma Cámara de representantes, con el rostro en alto ante la sociedad y los estamentos que representan.

Esta puesta en escena social de un debate del deber ser de la universidad el futuro y sus procesos de construcción debe ser abierto y democrático. Quizás sea cierto la afirmación que ronda en los pasillos de la desesperanza intelectual: La universidad es una democracia sin demócratas que forma demócratas.

Los consejos son los recintos donde los actores de la universidad valoran y anticipan las múltiples tendencias del conocimiento; de los impactos en la formación 
humanista, científica, tecnología y artística de las actuales y próximas generaciones. Existen acercamientos entre las fronteras de las ciencias, las tecnologías y las innovaciones donde un nuevo sentido a la transversalidad del conocimiento; nuevas profesiones brotan dese las alianzas científicas e inéditas tecnológicas, con profundizaciones disciplinarias interdisciplinarias, multidisciplinarias y transdiciplinarias; con rupturas, fases creativas y fases destructivas" que marca una nueva forma de percibir interpretar y transformar el universo en expansión e implosión y en forma simultanea abordar la investigación de la cotidianidad con la mayor rigurosidad teniendo como eje el impacto en el bienestar de la sociedad.

Es esencial vislumbrar los escenarios de la universidad del futuro de una época de transición, entre lo mecánico y lo vital, en un escenario conflictivo planetario; y la añoranza de una comunidad pastoril y donde la sociedad de conocimiento aún no se consolida como cuerpo capaz de convertirse en paradigma. En embargo, avizoramos la complejidad conceptual que deberán manejar las nuevas generaciones, que buscan la estabilidad en un universo en movimiento; sentimos pánico por lo que está por nacer que ha sido fruto del ingenio humano, materialización de su inteligencia e innovación trasformadora.

El conocimiento que nace, se construye y se de-construye desde las teorías dialécticas a partir del trabajo en todas sus formas; desde los materiales hasta las más intangibles llegarán en esta época a convertirse, en sí mismos, en paradigma de transformación o de alienación social. Por ejemplo, la revolución científico tecnológica que nos está llevando a la complejidad del genoma humano, sin duda trasformará los ciclos vitales, las formas de abordar la vida, la ingeniería genética, y surgirán variados escenarios, desconocidos actores, inéditos campos de conocimiento, diferentes conceptos del ser, valor, ética, amor, sociedad y vida como nunca la humanidad lo había sentido en búsqueda de si mismo.

Los actores de la universidad deberán propiciar el análisis sobre la desaparición de campo laborales que duraron por siglos: el conocimiento será el factor central del desarrollo de las sociedades y de sus mayores conflictos, surgirán nuevos ambientes de aprendizaje que transformar\{a la actual universidad; se pasará de una universidad que enseña a una universidad que aprende; de una universidad que replica a una universidad que investiga, de una universidad que cree a una universidad que crea; de una universidad rígida a una universidad flexible. Sera necesario anticipar la ciencias básicas; así como el surgimiento de una nueva complejidad en la interpretación de las ciencias sociales y humanas menos occidentalizadas y universales e integradas, según Emmanuel Walestein

Es fundamental prever un acercamiento cada vez más profundo entre las ciencias, las tecnologías y el arte y la articulación de estos avances en relación paradójica entre la infinita capacidad de manejo de conocimientos y la miniaturización de la complejidad del conocimiento temas que deberán ser del dominio de los actores 
universitarios de la sociedad colombiana y latinoamericana para nuestra articulación e interfecundación creativas

Es necesario que los actores universitarios valoren las visiones de las organizaciones responsables de producir conocimiento en la región. La universidad no es una organización solitaria del conocimiento, existen otras organizaciones que también lo producen, por tanto, socios en el camino de su transformación y con ellos hay necesidad de realizar alianzas estratégicas para su abordaje cooperativo quizás lleguemos a elementos comunes en las visiones con otras organizaciones que tienen que ver con la producción de innovaciones y partes de la visión que determinan su visión compartida, si es necesario revistaremos el futuro en palabra de Juanjo Gabiña de estas organizaciones que llamaremos del conocimiento. Lo anterior implica que la universidad como líder tendrá que impulsar con la mayor brevedad la configuración de una red de universidades articuladas a las redes de universidades Andinas, a la Asociación de Montevideo (AUGM) a universidades Centroamericanas y del Caribe, consejo superior de Universidades de Centroamérica (CSUCA) a universidades estadounidenses, mexicanas y canadienses, asiáticas árabes y africanas

En este escenario existe una crisis interesante, creativa relacionada con el relevo generacional, estamos frente a la oportunidad de entregar a la nuevas generaciones las llaves maestras de los escenarios que serán los adecuados para la transformación dela universidad, el desarrollo histórico de la universidad como constructo-teórico que perduró por siglos; es una crisis planetaria que estremece las estructuras añejas de la normatividad universitaria del derecho, que pretende regular el futuro maniatándolo a sus articulados que niegan la transformación de la universidad. La tensión en la transformación de las universidades parece centrarse en el dilema "construir el futuro de la universidad a partir de las normas o construir el futuro de la universidad a partir de la transformación del conocimiento"

\section{Reflexiones y propuestas}

- La transformación de la Universidad en palabras de Didriksson debe vivir en fase de transición caracterizada por episodios donde los símbolos la universidad tradicional se mantienen vivos y los símbolos de la nueva universidad productora de conocimientos, anuncian su fuerza naciente.

- Según Didriksson existen tres formas de transformar la universidad: la primera, se sustenta en la introducción de la otra estructura paralela autónoma; la nueva y la vieja conviven sin amancebarse. La segunda Forma, invita a colocar cargas explosivas en puntos estratégicos de la institución de tal forma que sus estructuras obsoletas se debiliten por efectos demostrativos de unidades academias totalmente nuevas, innovativas y exitosas, y la tercera, sería la innovación universitaria de una universidad del futuro que se mueve en todos sus procesos. Una que nace nueva en todas sus 
dimensiones. Es fundamental pensar en estructuras hibridas en un proceso de transición entre la sociedad industrial y la sociedad del conocimiento para el inicio de la transformación de la universidad, que requerirá una fuerte modernización en su infraestructura científico tecnológica y de formación de talentos humanos desde una visión humanística. El paso de la transición implica la presencia, aun de los rasgos de la universidad. La experiencia en la transformación de universidades demuestra que es un proceso, conflictivo, duro, extenuante, que debe ser acompañado por organismo y expertos nacionales e internaciones en la transformación de las universidades.

- Crear los espacios y ambientes para la construcción del nuevo proyecto de universidad, que convoque a todos los actores políticos, académicos, estudiantiles, económicos, sociales y culturales a su construcción, este proceso implica una transformación en las concepciones curriculares y en las formas organizacionales de la Universidad vista desde escenarios futuros. -A partir del nuevo proyecto de universidad se deben encontrar las alianzas estratégicas correspondientes y desarrollar los pactos que deben ser establecidos a través de relaciones que integren los ingredientes de la nueva sociedad del conocimiento. La universidad debe crear centros de estudios de sus contextos de acción con el propósito de analizar su pasado presente y futuro de los aspectos implicados con el desarrollo de sus escenarios. En la actualidad el espacio para la divergencia está congelado, la diversidad es frágil, la heterogeneidad se asocia con decadencia, la denuncia y la sospecha son productores de poder; está sola actitud compromete las nuevas formas de producción del conocimiento. Las tendencias curriculares del tercer milenio indican transformaciones hacia organizaciones en Redes, Escuelas y proyectos para concretar estrategias curriculares que se sustentan en la movilidad, la flexibilidad, la cooperación y en un sistema de créditos académicos, a través de cuatro etapas de formación: tecnológica, profesional, maestrías y doctorados; todas ellas atravesadas por ejes de formación transversal e investigación que le den unidad y coherencia a las nuevas estrategias de producción de conocimiento con niveles significativos de retorno social.

- La transformación de la Universidad implica propiciar reflexiones en torno a un nuevo modelo curricular donde la investigación tenga un escenario para la flexibilidad, la integralidad y la cooperación con el apoyo de las nuevas tecnologías de la información y la comunicación (NTIC), basado en redes académicas, y en una modernización de la plataforma científica tecnológica que promueve la formación de ciudadanos y de profesionales con criterios de calidad emanados de un diagnostico regional, en el contexto nacional e internacional y que promueva la articulación e integración de las funciones sustantivas, en el marco del cambio estructural general del sistema de 
educación superior nacional. En la nueva sociedad del conocimiento, los procesos de formación, generación difusión y aplicación del conocimiento, contaran con un alto grado de compromiso y retorno social y retomaran los principios básicos de la Declaración Mundial de la UNESCO: aprender a aprender, aprender a ser, aprender a hacer, aprender a emprender y aprender a respetar al otro y al entorno, y los valores institucionales de ética, solidaridad, responsabilidad, integridad, respeto a la diversidad y tolerancia. Los organismos mundiales tales como la UNESCO promueven escenarios de cooperación y para ellos, recomiendan que la universidad se comprometa en determinados cambios: Una organización académica interdisciplinarias que propicie la formación interinstitucional concertada y descentralización.

- El modelo se sustenta en una red que facilita la articulación de las funciones sustantivas de investigación y extensión en el proceso formativo, en la flexibilidad, y polivalencia y actualización de sus planes y programas, como también en la actualización de su personal académico, administrativo y directivo. De acuerdo con lo anterior, las estrategias curriculares de la universidad tienen a abordar los siguientes retos: Una educación centrada en el estudiante, que propicie la flexibilidad, la movilidad y que facilite la cooperación. La educación centrada en el aprendizaje ha sido planteada por la UNESCO con el propósito de facilitar que la educación superior se articule a la complejidad del conocimiento, a la diversidad de las estructuras sociales y a los nuevos métodos de enseñanza. Se enfatiza que se requiere pasar por un paradigma centrado en la enseñanza y la transmisión de conocimientos a otro centrado en el aprendizaje y el desarrollo de competencias trasferibles a contextos diferentes en el tiempo y en el espacio. Por lo anterior, los procesos de enseñanza- aprendizaje. Así como los programas de estudio de la Universidad Surcolombiana, estarán enfocados hacia el aprendizaje colectivo e interdisciplinario del conocimiento desde una perspectiva del desarrollo con valor agregado social.

- Con relación a las propuestas de la conferencia Mundial sobre Educación superior de la UNESCO relativas al futuro de la educación superior en el mundo, la Universidad promoverá la movilidad, la flexibilidad, la interdisciplinaridad. La transversalidad y la cooperación académica, entre sus facultades y escuelas a partir de las innovaciones en el sentido que ellas propician: La incorporación de nuevos métodos que propician en una adecuada relación entre la teoría y sus aplicaciones prácticas, lo que implica el desarrollo de conocimientos, pero también de las habilidades y destrezas requeridas para facilitar dicha aplicación en currículos por ciclos y polivalentes. -La formación permanente a través de la vida con el fomento de la creatividad y del espíritu de iniciativa, con el desarrollo integral de las capacidades cognoscitivas, afectivas, sociales y de convivencia, donde 
primera el espíritu crítico y el sentido de responsabilidad social. La formación del más alto nivel de calidad, tanto en el plano técnico profesional y científico, como en el plano de la formación de la nueva ciudadanía. La producción científica, artística, humanística, tecnológica e innovación a través de artículos científicos, libros, textos, software, hardware, indexada y patentada con reconocimiento económico para la institución y para sus creadores. De acuerdo a lo anterior, la universidad deberá aprovechar plenamente las nuevas tecnologías de la información y la comunicación (NTIC), que hoy impulsan el desarrollo de nuevas experiencias de aprendizaje. En este escenario, los grandes principios orientadores del quehacer académico de la Universidad implican: una visión integral, una educación centrada en el estudiante, una educación pertinente y socialmente comprometida y una educación polivalente.

- La educación integral debe reflejarse en la visión y misión de la Universidad. Es un compromiso histórico, vital y permanente con la construcción de una sociedad justa y equitativa, pero también, en la formación de un ser humano integral, ético y solidario, en una educación con responsabilidad con la sociedad que además de ser activa y continuo, y deberá asegurar la calidad, pertinencia, cobertura y la democracia en sus acciones. La educación centrada en el estudiante significa promover la construcción de nuevos ambientes para la formación individualizada y colectivizada del estudiante, en la cual, el propio estudiante, pueda tomar decisiones en cuanto a los ritmos, modos y tiempos en que cursará los programas educativos de la Universidad, de acuerdo a sus necesidades, características, antecedentes académicos y posibilidades de dedicación, generando así un sentido de responsabilidad, libertad y mayor autonomía. Así mismo, una educación centrada en el estudiante estará orientada por métodos y técnicas didáctico pedagogías que pongan en el centro de la atención, precisamente, al sujeto que aprende.

- La educación pertinente y socialmente comprometida es un componen de la formación integral. Hay pertinencia cuando el precios educativo se proyecta a la realidad del entorno y el momento histórico y cultural, cuando considera para su actuar académico las prioridades, exigencias y necesidades de la comunidades de aprender a aprender, la flexibilidad, la transversalidad y la movilidad en las condiciones cambiantes del entorno, el trabajo en equipo, la criticidad constructiva y reflexiva frente a las normas y saberes de una disciplina (s), cuando se fomenta la capacidad de auto-actualizarse permanentemente, cuando se recrea el conocimiento al servicio de la sociedad, cuando el profesional es respetuoso y solidario con el desarrollo social y cultural. Todo lo anterior, conduce a la formación de individuos comprometidos con el bienestar de la sociedad y el entorno y con una actitud 
de respeto al otro y a la democracia, en este sentido, es pertinente cuando se aproxime a la características de entorno natural y humano, al mundo del trabajo, al contexto social, económico y cultural, a los desarrollos científicos tecnológicos de hoy, al mundo y la cultura con las cuales contemporizamos. En estos términos, la pertinencia busca relacionar el currículo con los fines educativos, las necesidades del medio y del desarrollo social e individual. Es el compromiso con el desarrollo de la región y del país, mediante una formación una alta pertinencia profesional. Estos elementos deben propiciar procesos curriculares que fomenten a los profesores y estudiantes de la Universidad Surcolombiana, acciones académicas, personales y profesionales trascendentes.

- La educación polivalente le permite a la Universidad incorporar desde su diseño, los contenidos culturales necesarios para que le sea posible, a los egresados encontrar diversas formas de práctica profesional. Esta polivalencia será posible en la medida en la que los contenidos los programas educativos enfaticen en los aspectos básicos de la formación; así como en el desarrollo de competencias para la vida que harán parte de una etapa de formación común a todos los estudiantes de la universidad; y, competencias profesionales que serán parte de la etapa de formación profesional de todo plan de estudios.

- La educación es competente cuando este principio se refiere a la capacidad idónea, construida en las aulas de la Universidad, para que los egresados solucionen los problemas que corresponden a la formación profesional. Ellos implica que los contenidos de los planes de estudio consideran no solamente los conocimientos teóricos propios de una formación, sino que también enfaticen en la identificación de las vías de aplicación de esos conocimientos y en el desarrollo de las habilidades y destrezas necesarias para ellos. Al mismo tiempo, se deberá considerar la inclusión como estrategia de aprendizaje; la investigación, el análisis y estudio de casos, así como la solución de problemas, entre otros.

A continuación se expondrán algunas estrategias puntuales para ampliar la cobertura académica de la Universidad

\subsection{Ampliar la cobertura de días y horarios académicos}

Ampliar la cobertura de los horarios de la Universidad de domingo a domingo y entre las cinco de la mañana y las once de la noche. Las tendencias universales en el manejo de los horarios y plantas físicas, indican el surgimiento de una universidad de veinticuatro horas con el máximo disfrute de sus espacios académicos: profesores, clases, aulas, investigaciones, laboratorios, asesorías, consultorías, talleres, bibliotecas, gimnasio, pistas, piscinas y auditorios. Esta propuesta permita ampliar el ingreso de los estudiantes, tanto a las carreras que la universidad ofrece 
en la actualidad, como en las nuevas carreras que requiere la complejidad de la nueva sociedad del conocimiento, así como las riquezas potencias y las necesidades de la región. Este importante enfatizar que no es una estrategia administrativa para crear carreras nocturnas, sino brindar las condiciones para que los estudiantes tengan una mayor movilidad en el acceso a las universidades de domingo a domingo, en un horario flexible que varía entre las horas propuestas y que les permite combinar relaciones teóricas del conocimiento con verificaciones sociales de su aplicación; necesidades laborales con oportunidades academias de los estudiantes que teniendo un puntaje ICFES alto, no pueden acceder a la universidad; o de aquellos estudiantes que por compromisos y condiciones familiares, sociales, o culturales, no pueden disfrutar de una movilidad curricular en facultades, escuelas, proyectos, horarios y disciplinas flexibles.

2.2. Ofrecer una ampliación en las carreras que ofrece la universidad con salidas laterales.

Esta idea no es novedosa en sí, pues varias universidades la orecen el mundo inclusive en Colombia. La idea consiste en establecer un tronco común afín a una facultad cualquiera; para el ejercicio se propone la facultad de ingeniera en la Universidad Surcolombiana. Este tronco común corresponde a los dominios que en las disciplinas debe manejar un ingeniero. Desde la plataforma se ofrece en convenios con instituciones de la región, como el Servicio Nacional de aprendizaje SENA, Institutos técnicos Superiores, Institutos Técnicos Agropecuarios, INEM, con instituciones de nivel superior, del orden, nacional e internacional, -instituciones debidamente acreditadas- ante el ICFES, en caso de las nacionales o reconocidas en los respectivos padrones de excelencia en sus países, entre otros, formación universitaria desde los niveles de técnicos u Auxiliares en laboratorios, hasta doctorados y en próximas décadas postdoctorados. Esta propuesta curricular propicia salidas alternas, o laterales, "no es como sistema terminal cerrado", el estudiante puede reingresar a su carrera, o a una carrera afín, (recuérdese que existe un tronco común de formación) y continuar hacia la formación como Tecnólogo, Ingeniero, Magister y Doctor. Desde esta perspectiva estaríamos abordando el diseño de una currícula polifuncional. Esta propuesta permite combinar la formación académica, con el ejercicio laboral, sin embargo, si el estudiante quiere realizar su formación continua, lo puede hacer desde tecnólogo hasta doctorado, sin interrupciones y reingresar cuando lo desee, desde esta perspectiva se aborda uno de los retos planteados por UNESCO en parís para las universidades del tercer milenio, "una educación a través de la vida y para la vida."

Si se aspira por lo menos, a procesos de diaculturalidad entre las naciones, es necesario en esta tensión entre globalidad, regionalidad y localidad establecer parámetros de comunicación entre culturas. Desde este escenario es loable una licenciatura en idioma-ingles, que se les permita a las nuevas generaciones latinoamericanas, un dialogo de culturas, lo que implica que el aprendizaje del 
inglés, desborda los límites, del solo dominio de las cuatro habilidades comunicativas, para adentrarse en el dominio de otra cultura en sus vertientes históricas, sociales, políticas, economías, científicas, tecnológicas y artísticas. Sin embargo este aprendizaje cultural de los idiomas en harás de las normas vigentes no puede enjaularse en la Facultad de Educación. El dominio cultural de un idioma es una formación polivalente que le permite a cualquier estudiante del mundo desde un tronco común curricular alcanzar dominios como la traducción, composición, interpretación, representación y transformación de textos, roles y profesionales del siglo XXI que deberán manejar otros idiomas de acuerdo a los avances que estos países hallan desarrollados en la perfección de sus estatutos científicos. Este proceso por su complejidad reposa en el dominio de la cultura nativa, de lo contrario sería muy complicado hablar de procesos de diaculturalidad, América latina tiene un coloso hermano y desconocido Brasil, sin embargo poco interés hemos demostrado por el portugués, siendo nuestro socio más cercano dada la riqueza de la biodiversidad amazónica nuestro escenario de mayor potencialidad vía siglo XXI.

\subsection{Ofrecer una diversidad de espacios para trabajos con la investigación}

Siguiendo los estudios de Gibbons nos enfrentamos a una "disolución de las fronteras entre las ciencias, las tecnologías y el arte, se está pasando de los trabajos donde predominaba la disciplinariedad, Modo 1, a estudios donde comienza a tomar fuerza la interdisciplinariedad, la multidisciplinariedad la trasdisciplinaridad, Modo 2 en la producción del conocimiento al poder relacionar las cuatro funciones modernas de la universidad: investigación, innovación, formación y socialización. Esta propuesta se acompaña con una modernización de la infraestructura científica, tecnológica, conceptual y en las nuevas tecnológicas de la comunicación y de la información; así como de la plataforma internet.

2.4. Modernización de la infraestructura científica, tecnológica, conceptual y la plataforma de la universidad.

Para zarpar no es suficiente conocer los posibles escenarios futuros hay que auscultar la complejidad del escenario donde vamos a navegar. Es necesario revisar en el presente, y desde la sincronía el equipaje del conocimiento acumulado a través de la investigación, una mirada diacrónica del estado del arte conceptual acumulado, una valoración de los talentos humanos, de la infraestructura científica tecnológica.

Teniendo un horizonte futuro a donde ir, visión, misión, principios y macroproyectos con sus funciones universitarias: formación ( proyectos curriculares y títulos); investigación (proyectos de investigación, publicaciones) innovación ( experimentación y patentes) y socialización ( exposiciones y proyectos educación permanente); revisado nuestro equipaje real y potencial, diseñaremos las rutas de navegación, prepararemos la bitácora, a los actores viajeros, la infraestructura y la plataforma de comunicaciones interconectada, moderna, ágil e instantánea; 
organizaremos nuestro observatorio universitario con veedurías e iniciaremos el viaje que implica un compromiso real de la sociedad y de su Universidad.

Para ellos es fundamental estar modernizados con las nuevas tecnologías de la información y la comunicación (NTIC); así como contar con una sólida infraestructura científica, tecnológica y artística.

\section{Referencias}

DIDRIKSSON T, Axel. Serie de conferencias sobre la universidad del futuro y sus textos sobre el mismo tema, Centro de estudios Universitarios CESU, UNAM, México, 1998 - 1999.

GABIÑA, Juanjo. El futuro revistado. LA reflexión prospectiva como arma de estrategia de decisión. Alfomega Marcombo, 1998.

MACHADO, Ana Luisa. Directora OREALC UNESCO, Ana Maria Corvalán Coordinadora SIRIOREALC. "La educación en América Latina y el CaribeProspectiva al 2020. Rio de janeiro, 1999.

TUNERMANN, Carlos. La educación superior en el tercer milenio UNESCO. Caracas- Venezuela, 1999. 